\title{
Undergraduates' awareness of White and male privilege in STEM
}

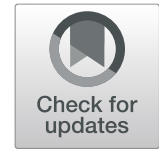

Melissa Dancy ${ }^{1 *} \mathbb{D}$, Katherine Rainey ${ }^{1}$, Elizabeth Stearns ${ }^{2}$, Roslyn Mickelson ${ }^{2}$ and Stephanie Moller ${ }^{2}$

\begin{abstract}
Background: It is well-documented that experiences in STEM courses for women and students of color are different from the experiences of White men. As part of a larger interview study, 183 college seniors from diverse gender and race backgrounds were asked their thoughts on whether the experience of being a STEM major was different for people of different races and genders. We use a framework of "science as White property", derived from critical race theory, to frame this study and results.

Results: White men were largely unaware of any impact of race or gender. In contrast, women of color overwhelmingly report, consistent with results from a large body of prior research, that both race and gender impact their experiences as STEM majors. Students who acknowledged race and gender impacts did not always attribute these impacts to cultural or systemic factors (i.e., some reported women are underrepresented because they are less interested in STEM rather than a structural reason). Impacts identified that were attributable to systemic factors included impacts related to being a demographic minority (i.e., intimidation, feeling out of place, feeling pressure to work harder) and/or discrimination (i.e., job discrimination, bias against women or people of color and cultural assumptions implying the superiority of White people and men). A small number of students (mostly White women) stated that women or people of color benefit from their underrepresented status, often attributing this benefit to a perception of extra encouragement and opportunities. A common theme across categorizations was that women and students of color work harder than men and White people either because they are perceived to be harder workers or as a response to the sexism and racism they encounter.

Conclusions: We found that White men are largely unaware of the impacts of race or gender on the pursuit of a STEM degree. Additionally, with the exception of women of color, students are less likely to perceive race as having an impact on the experiences of students than gender. We conclude with a discussion of implications for future work related to gender and race representation in STEM.
\end{abstract}

Keywords: Race, Gender, Undergraduates, Qualitative research

\section{Introduction}

White men have historically had opportunities in STEM that have been denied to other students. To date, STEM students are generally overrepresented in terms of gender by men and, with respect to race/ethnicity, by White students and students from certain Asian ethnicities (National Science Foundation and National Center for Science and Engineering Statistics, 2019). Many observers have raised concerns about

\footnotetext{
* Correspondence: melissa.dancy@gmail.com

'Department of Physics, University of Colorado Boulder, UCB390, Boulder, CO 80309-0390, USA

Full list of author information is available at the end of the article
}

these enrollment patterns because of the waste of talent among historically excluded populations and issues of distributive justice related to STEM careers and social mobility (Hill, Corbett and St Rose, 2010). Despite decades of frequently well-funded efforts to achieve greater gender and race/ethnic equity in STEM majors, on balance, most fields have seen only minimal improvements (National Science Foundation and National Center for Science and Engineering Statistics, 2019).

In an effort to understand more about inequity in STEM, we engaged in a large-scale interview study of undergraduates in STEM. Here, we use a conception of 
"science as White property," (Moore Mensah and Jackson, 2018) adapted from of critical race theory (CRT). We combine the science as White property concept with an approach that also emphasizes the intersections of race/ ethnicity and gender to frame the experiences of students in STEM classes, forming a conception of science as White male property. Further details and additional findings from these interviews are reported elsewhere (Mickelson, Parker, Stearns, Moller and Dancy, 2015; Moller et al., 2015; Rainey, Dancy, Mickelson, Stearns and Moller, 2018; Rainey, Dancy, Mickelson, Stearns and Moller, 2019; Stearns et al., 2019). In this article, we present students' views regarding racial and gender differences in experiences of students in STEM. This analysis includes both the views of students from marginalized groups and those from groups considered to hold privilege, i.e., White males. There are few published reports of the perceptions of students from privileged groups in STEM. In particular, it is unusual to see distinctions made between views of those from marginalized groups and those from more privileged groups, as we do in this article.

We highlight important distinctions in perceptions of racialized and gendered differences in STEM classroom experiences identified in the interviews because disparities in these views may help us understand the persistent patterns of privilege within STEM. Many students saw no race or gender differences in STEM experiences. Among those who did, explanations ranged from differences in people to explanations embedded in cultural systems. Students' own race and gender related to if and how they perceived differences in STEM experiences. We conclude with a discussion of our findings' implications.

\section{Overview of study}

The study presented here was part of a larger study, the Roots of STEM Success Project (https://pages.uncc.edu/ rootsofstem/) which involved interviews with selfselected students, all of whom were enrolled in one of the 16 campuses of the University of North Carolina system. Here, we report on analysis of two specific groups. The first group ( $n=138$ ) was composed of STEM majors in their senior year (majors). The second group ( $n=$ 45) consisted of seniors who had started but dropped a STEM major and were no longer pursuing STEM (leavers). Each interview was approximately $1 \mathrm{~h}$ long, took place over phone, and included many questions designed to elicit students' accounts of the influences and experiences that encouraged them toward or discouraged them from pursuing a STEM major. When possible, the race and gender of the interviewer was matched to the race and gender of the participant. In this paper, we focus on two questions (described in detail later) asked in the interview probing for their perceptions of race and gender impacts in STEM.

\section{Conceptual framework}

The broader conception of "Whiteness as property" stems from critical race theory and its assertions that White people can claim some tangible outcomes as their own, thus denying others access to that property (Annamma, 2015; Haney-López, 2006; Harris, 1995). Building on Ladson and G., and Tate, W.'s (1995) work that applied that concept to education, Moore Mensah and Jackson (2018) point out some of the many ways that patterns of privilege within STEM reflect power inequities that demonstrate Whiteness as property. As they note, "the right to use and enjoyment of sciencewhat science looks like, who engages in science, and what science is for" (p. 3, original italics) has historically excluded the participation and representation of women and people of color and been reserved for Whites. In other words, the inclusion of only White contributions to and participation in science generates "science as White property."

\section{Intersectionality}

We amend Moore Mensah and Jackson's notion of science as White property by incorporating an intersectional approach and note that science has been a form of White male property, in that it is a form of privilege from which White women and people of color have historically been excluded. Thus, the analysis presented in this paper takes an intersectional approach. Intersectionality refers to the idea that aspects of one's identity (e.g., race, gender, class, sexual orientation) are not mutually exclusive but instead interact to construct one's identity (Collins, 2015; Crenshaw, 1990). Considering race and gender as single axes of identity without analysis of the intersections of these statuses can lead to the erasure of some identities, such as those of women of color (Bowleg, 2008; Crenshaw, 1990) whose experiences are more than that of women generally and/or of people of color generally, but rather are uniquely influenced by their multiple identities and the multiple systems of oppression that impact them. In this study, we look at two axes of identity-race and gender-and the intersections of these identities: women of color, men of color, White women, and White men. This approach allows us to understand not just the impact of race or gender but also the impact of the intersection of those identities on how our interviewees perceive the experience of being a college STEM major.

Though there are other axes beyond race and gender that could be considered, these are the only axes of identity we recorded for this study. We acknowledge this limitation but believe that this partial-intersectional approach will help avoid the erasure of some students' complex identities, such as those of women of color, by allowing the reader to see both gender and race analyses. 


\section{Background research}

Access to STEM for women and people of color has been a concern for several decades. Reviews summarizing the vast literature regarding equity issues in STEM can be found elsewhere (Committee, 2011; Hill et al., 2010; Ong, Wright, Espinosa and Orfield, 2011; Rugheimer, 2019). Though much is known about access issues in STEM, less is known about perceptions of dominant and underrepresented groups regarding race and gender dynamics in STEM. Below, we highlight what is known about the experiences of women and people of color, as well as general trends regarding perceptions of race and gender in the broader context of the USA. We use these findings as a grounding for our investigation of perceptions of race and gender in STEM-specific domains.

\section{The experience of being a STEM student is different for people of different races and genders}

We ask students in our study if the experience of being a STEM major varies for different genders and races. The preponderance of extant research on the question strongly suggests that the answer to both of these questions is yes. Students are significantly impacted by both their race and gender as they pursue STEM. For example, research indicates that both women and people of color are more likely to report discrimination, microaggressions, and harassment in STEM (Rugheimer, 2019) and are less likely to feel they belong (Fisher et al., 2019; Rainey et al., 2018), findings that are all consistent with the notion of science as White male property. These experiences have been found to affect the physical and mental health of women and people of color, their ability to thrive in STEM fields, and their willingness and ability to continue in STEM (Fisher et al., 2019; Hill et al., 2010; Rugheimer, 2019). It is important to note that the question we ask of students in our interviews is not their opinion on these matters but their perceived experiences with racialized or gendered issues in STEM classrooms. By the time students are seniors in college, they are highly likely to have had the opportunity to experience and or witness impacts of both racism and sexism. The question we take up in this analysis is as follows: to what extent are students aware of these impacts and how does awareness differ by demographic characteristics of the interviewee?

\section{Men are less likely to perceive sexism; white people are less likely to perceive racism}

Generally, studies of perceptions of the impacts of race and gender in STEM report on the perceptions of a marginalized group only (i.e., women's perceptions of gender discrimination in STEM). Studies comparing the perceptions of the same environment across the genders and races are so rare we have had difficulty finding any in the academic literature. The best data we have found comparing perceptions comes from polls conducted by the Pew Research Center. These polls consistently show males perceiving fewer gender impacts than women and White people perceiving fewer race impacts than people of color.

For example, a recent poll of STEM professionals about their workplace environment (Funk and Parker, 2019) reports consistent gaps between the perceptions of discrimination between women and men. The researchers found that $38 \%$ of women in male-dominated STEM fields felt women are usually treated fairly in their workplace in opportunities for promotion and advancement compared to $78 \%$ of men. Likewise, they report a gap among races. In response to a question about fairness in promotion and advancement for Black people, $37 \%$ of Black people reported fairness compared to $75 \%$ of White people.

The same poll also found that reports of discrimination in STEM are higher than in non-STEM fields. Women in male-dominated STEM fields report experiencing discrimination at work more than women in non-STEM jobs (50\% vs. $41 \%)$ and Black people in STEM fields report experiences of workplace discrimination due to race more than Black people in non-STEM jobs $(62 \%$ vs. $50 \%)$.

\section{Some members of privileged groups believe they are the disadvantaged group}

In addition to a pattern of evidence that privileged groups fail to recognize their own privilege, there are also indications that, despite evidence to the contrary, privileged groups may believe they themselves are the disadvantaged group. This is presumably in reaction to policies and practices like affirmative action that acknowledge and attempt to address inequity. For example, Norton and Sommers (2011) report on a recent study demonstrating that on average White people in the USA believe anti-White bias is more prevalent today than anti-Black bias. They also offer evidence that White people believe reverse racism is increasing over time. In general, these perceptions may be the result of a lack of contact with members of other groups or because White people feel threatened by diverse contexts and wish to protect their position (Tropp and Barlow, 2018). Perceived threats may also be psychological, including the suggestion that their position has not been the result of their own merit or that they are part of a group that has unfair advantages. As Knowles, Lowery, Chow and Unzueta (2014) argue, when perceiving these threats, White people can respond in one of three ways-denying discrimination, distancing their self-concepts from the "White" label, or attempting to dismantle forms of discrimination. 
Members of marginalized groups do not always acknowledge their own marginalization

While research consistently finds that members of marginalized groups are more likely to recognize the impacts of their demographic status on life chances than those of the privileged group, studies, such as the Pew Research Center poll described above (Funk \& Parker, 2019) also find a significant proportion of the marginalized group that does not report an experience with discrimination. This discrepancy has been noted and theorized by researchers across varying fields.

One explanation for the disconnect between perception and experiences is the denial of personal disadvantage. In Crosby (1984) on the denial of personal disadvantage, she identifies the discrepancy between women's acknowledgment of discrimination in general but denial that they are personally discriminated against, despite evidence to the contrary. Crosby proposes two barriers preventing women from acknowledging their own disadvantage. First, she suggests the cognitive bias associated with analysis of large numbers vs. individual cases obscures discrimination of an individual. Secondly, she suggests the emotional discomfort people experience when confronting their own victimization as a barrier for acknowledging disadvantage.

Another notable explanation for the failure of marginalized groups to recognize their disadvantage is system justification theory. Jost and Hunyady (2003) review system justification theory, which offers insights into why people justify the status quo even when it is against their own self-interest. They suggest people have a tendency to support and defend existing social structures due to a need to view themselves and their group positively. Members of marginalized groups engage in system justifying behavior because such engagement serves a palliative function to minimize the harm they encounter in circumstances they cannot change.

In summary, the research literature indicates that racism and sexism are pervasive in STEM, reflecting the idea of science as White male property. White people tend to be less aware of racism than people of color and men tend to be less aware of sexism than women, and some members of both marginalized groups deny their marginalized status. One question we are able to investigate through comparative analysis of gender-by-race perceptions of racism and sexism in STEM is whether the intersection of students' race and gender influences the likelihood of their recognizing or denying privilege or marginalization.

We employ a number of terms that are frequently interpreted colloquially in ways that may differ from our use here. Below is a list of terms and how we use each concept in this manuscript.
Privilege refers to a set of unearned advantages, such as access to resources or social power, available only to certain people because of their membership in a social group (NCCJ, 2018). Privilege can exist among many dimensions (race, gender, religion, age, sexual orientation, etc.) and varies between societies and cultures. Having privilege does not mean someone did not have to work for their success or never experienced adversity, nor does it mean someone is intentionally oppressing people of other groups. Instead, it refers to unintended systemic advantages certain groups experience that simultaneously disadvantage others without these ascribed characteristics.

Prejudice is the conscious or unconscious thoughts, beliefs, assumptions, and cultural stereotypes of individuals about the superiority or inferiority of certain groups that underlie discrimination for or against people in those groups. Under this definition, prejudice is an individual's set of beliefs about people who share characteristics of the "other" group (or their own favored group) and may be the basis of discriminatory behaviors.

Sexism and racism refer to systems of disadvantage based on gender and race. We use the prejudice plus power definition of these terms in this paper. Sexism and racism are different from prejudice in that they go beyond simple bias by individuals and are embedded in the power structures of society. We emphasize here that one can uphold sexist and racist norms and values unintentionally; it is possible to perpetuate racist and sexist ideologies without holding any personal beliefs about the inferiority of other races or genders, i.e., without being prejudiced. For an in-depth discussion about this phenomenon with regard to race, we suggest "Racism without racists: colorblind racism and the persistence of racial inequality in the United States," by Bonilla-Silva (2006).

We offer the following as an example that illustrates these terms. An individual man holding the belief that women are less mathematically able than men is prejudice. Utilizing standardized tests constructed with items that overestimate the mathematical ability of men while underestimating the mathematical ability of women to determine admission to college or graduate school is sexism. This thereby confers privilege onto men as they are able to gain admission with less merit. For an example of this phenomenon, see Miller, Zwickl, Posselt, Silvestrini and Hodapp (2019).

Marginalization is tied to structures of privilege and power. The concept refers to the relegation of people from certain groups to the margins of a society's organizations, institutions, and cultural system by denying them an active voice, identity, or place within the given social context (Syracuse University, 2018). People from these excluded groups can be described as marginalized in these contexts. 


\section{Methods}

\section{Description of data}

This article is part of a larger study which was, in part, designed to understand the lack of representation of certain demographic groups in STEM. Participants in the study were self-selected college seniors who attended one of the 16 public state four-year universities in North Carolina (NC). Interviewees were recruited using a survey sent through their university emails.

Due to the study's central focus on underrepresentation in STEM, women and students of color were oversampled for the interviews. Therefore, the sample is not representative of STEM in either race or gender. Racial demographics of the interview sample are shown in Fig. 1 and closely match the demographics of NC. Women were oversampled: $66 \%$ of our sample were women; non-binary gender options were not provided as options on the demographic form. Both race and gender identities are based on the self-reports of the participants in the initial recruiting survey. Of the students interviewed $41 \%$ were in majors that were categorized as female-dominated (i.e., biology and related fields), $20 \%$ were in gender neutral majors (i.e., math, agricultural science, chemistry), and $39 \%$ were in male-dominated majors (i.e., engineering, physics). We note that academic achievement was not a selection factor for interviewees. All of the interviewed students were slated to graduate with a college degree within the academic year in which the interview was conducted.

We combine students from all STEM majors and do not attempt to separate out responses based on field of STEM. The number of students in each category if we disaggregated by even broad STEM areas would be too

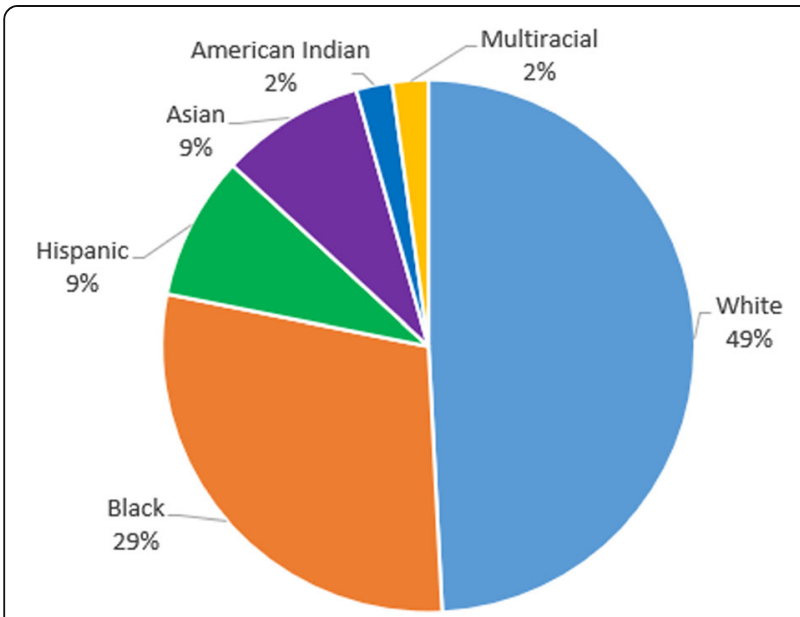

Fig. 1 Demographics of interviewed students. Demographics deviated from those in STEM but closely match the demographics of the geographic location in which interviews were conducted. Note that White and Black students made up the two largest demographic groups small to carry meaning in comparison. We do not claim that there are no differences by major field.

We interviewed students using a semi-structured interview protocol, which included questions designed to elicit the story of students' history with STEM. All interviews were audio recorded and transcribed. Here, we present an analysis of responses students gave to two specific questions asked near the end of the interview:

1. Is the experience of being a \{STEM major\} major different for people of different genders?

2. Is the experience of being a \{STEM major\} major different for people of different races?

Here, \{STEM major\} refers to the STEM major chosen or dropped by interviewees such as biology or chemistry. We analyzed students' responses using an iterative coding process facilitated in part by the qualitative analysis software Nvivo. Two of the authors, Dancy and Rainey, independently coded a subset of responses identifying themes and then compared. In this first pass we identified ideas students suggested as impacts and sought to collect under common labels. We then collapsed, expanded, and refined the identified ideas through a collaborative process. We then independently coded another subset of interviews and again compared, further collapsing, expanding, and defining codes. Disagreements in coding were discussed until full agreement was reached. Through this repeating process, we eventually reached a coding scheme in which the majority of responses could be coded. We then collapsed our codes under three broad categories descriptive of the source of the impact. These codes and categories are summarized in Table 1 and elaborated on in the presentation of results.

At the broadest level, responses can be categorized as either, "notices gender/race differences" or "doesn't notice gender/race differences." If a respondent indicated any difference in impact, we coded them as noticing a difference. A small fraction of the "notices a difference" responses indicated a belief that men or students of color benefited based on gender/race. We report such responses separately. For those responses coded as "notices differences," we then divided them into those which allude to sexism/racism (see definition of use of these terms above) and those which did not (difference was attributable to differences in genders/races and not systemic effects). For those coded as "notices differences," it was possible for a response to be coded in multiple categories if respondents expressed multiple beliefs. We found responses coded as identifying sexism/racism impacts fall in three main categories: impacts due to being a minority, impacts due to discrimination, and (for race only) 
Table 1 Coding scheme for gender and race differences analysis

\begin{tabular}{|c|c|c|c|}
\hline Doesn't notice & \multicolumn{3}{|c|}{ Not aware of a gender/race impact } \\
\hline \multirow[t]{5}{*}{ Notices differences } & Not sexism/racism & $\begin{array}{l}\text { Impacts due to differences in individuals not attributed to } \\
\text { systemic factors }\end{array}$ & $\begin{array}{l}\text { - Men and some races are more interested in } \\
\text { or value science more } \\
\text { - Women willingly work harder than men }\end{array}$ \\
\hline & \multirow[t]{3}{*}{ Sexism/racism } & Impacts due to underrepresentation & $\begin{array}{l}\text { - Intimidation } \\
\text { - Pressure to work harder } \\
\text { - Feeling out of place }\end{array}$ \\
\hline & & Impacts due to discrimination & $\begin{array}{l}\text { - Impacts for employment } \\
\text { - Bias against women/people of color }\end{array}$ \\
\hline & & \multicolumn{2}{|l|}{ Students of color lack social or cultural capital } \\
\hline & Differential benefits & \multicolumn{2}{|c|}{ Impacts due to advantages women and students of color receive because of their gender and/or race } \\
\hline
\end{tabular}

impacts of what we refer to as social capital. Table 1 summarizes these categories.

\section{Data analysis}

We conducted analyses separately for student responses about gender and race differences. The total number $(N)$ of respondents for both the gender and race analysis are shown in Table 2 below. For reporting, we divide race into students of color and White students. We do this because reporting results by specific race/ethnicity (i.e., Black, Asian, Hispanic, mixed-race) results in sample sizes too small for useful comparisons. Additionally, when we look at data from students of color, we find patterns across racial groups that are similar and distinctly different from White students. Finally, separating out by White students and students of color essentially divides the groups into those of racial privilege (White students) and those of non-privilege (students of color), which are essential identities relative to questions about racial impacts. We note that while Asian people are generally not underrepresented in STEM, calling into question their inclusion in the non-privileged group, there is ample data suggesting their experiences in STEM are more aligned with people of color than White people (i.e., they do not hold the same privileged status as White people). This is especially true for Asian women (Gee and Peck, 2017; Williams, Li, Rincon and Finn, 2016).

Table 2 Gender and race demographics of respondents coded for gender and race analysis

\begin{tabular}{llll}
\hline & & $\begin{array}{l}\text { Students } \\
\text { of color }\end{array}$ & $\begin{array}{l}\text { White } \\
\text { students }\end{array}$ \\
\hline Gender analysis respondents & Women & 53 & 53 \\
& Men & 31 & 32 \\
Race analysis respondents & Women & 56 & 54 \\
& Men & 32 & 30 \\
\hline
\end{tabular}

We report analysis from 183 total interviews. Due to variations in comprehensiveness of individual interviews (a question may have been deemed uncodable for various reasons), the sample size is different for each analysis: $N=169$ for gender and $N=172$ for race.

\section{Results}

In this section, we describe in detail respondents' responses to our two motivating questions. We also provide overall frequencies for the broad categories found in student responses.

\section{Finding one: Some students do not perceive any race and/or gender differences in STEM experiences}

If a response was coded as "doesn't notice gender differences" or "doesn't notice race differences," the respondent explicitly said there was no difference in the STEM experience for students of different genders or races or they stated they were unsure if there were differences. Many of these responses were of the form "I don't think so" or "not really."

Several respondents cited equal academic responsibilities for all students as an explanation for the lack of gender and race differences:

No I don't think it is. I think they're the same. Same course load, I don't think they get it any easier or any harder.-Black woman, electrical and computer engineering major

Also common was an appeal to the objective nature of science to justify a lack of impact relative to race or gender.

I feel like especially in the world of science we are being kind of objective about who we are and what we are doing, I think we are kind of on the same path.-White man, environmental science major

While most respondents in this category gave explicit responses about a lack of gender and race differences, 
some simply said they were unsure and did not commit to an answer. For example, a White woman majoring in biology stated "No I don't think so. I mean I couldn't imagine how it would be" and a White man majoring in aerospace engineering said "I don't know. It's kind of hard to say for me because I don't have any experience."

Distributions of respondents from each demographic group are shown in Fig. 2. White men were much more likely than other groups to deny gender and race differences for students in STEM and women of color were the least likely. Except for women of color, students were less likely to report perceiving an impact due to race than gender. Respondents coded in this broad category of "doesn't notice differences" were not coded for any of the subsequent broad categories which required them to identify a gender or race impact.

Finding two: Some students notice gender and/or race differences in STEM experiences and attribute them to individual characteristics, not sexism/racism

Some students indicated that they perceived differences in STEM students' experiences related to gender or race, yet they did not attribute these differences to any cultural or structural systems in which STEM education takes place. We coded statements in which differences were noted based on individual characteristics that were not directly or reasonably inferred to be attributed to systemic causes as "Individual Differences." Figure 3 shows the distribution of responses in this category. Under this broad category student responses were categorized into various theme groups, each of which are described below for both gender and race.

Differences in individual characteristics of men and women Some respondents described individual characteristics of men and women as the source of women's different experiences in STEM, without inference to these characteristics being driven by sexism. Below we describe the two most common individual characteristics cited. White men were the most likely to see individual differences as the key factor in differing experiences of the genders.

Belief that women are not as interested in science as men After being asked if there were gender differences in their major, some students (nearly all men) noted the gender imbalance and offered women's ostensible lack of interest in science as an explanation. For example, as a White man majoring in electrical engineering explained, "We do an open house and every time I talk to girls, with the exception of one out of the hundreds of families I have seen walk by me, only one seemed interested in electrical engineering and she knew that is exactly what she wanted to do. Others seemed to be afraid of the subject, they are probably afraid it would be too challenging or hard, but I suppose they probably don't know much about the major."

Women willingly work harder than men Some students (nearly all women) proposed that gender differences were due to effort expenditures. That is, the idea that women work harder than men. In order to be included in this category the response appeared to be a quality of the gender and not attributed to a systemic cause (i.e., women work harder because of discrimination). Those citing that women work harder due to a systemic cause are discussed later in another category.

For example, a White woman majoring in biotechnology said "What I've seen is most of the girls at our school are a lot more willing to put in the extra work to study versus some of the guys." In many cases, this

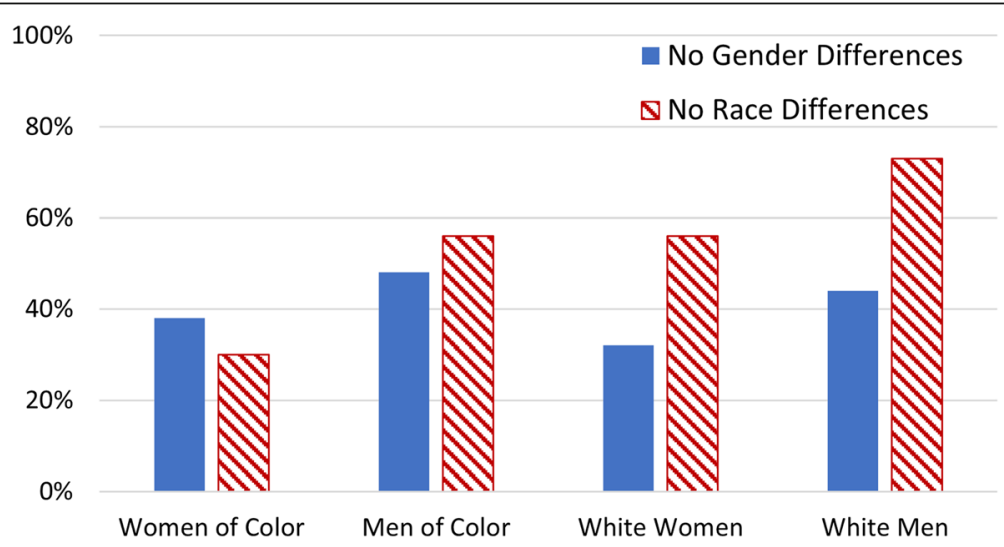

Fig. 2 Percent of respondents who did not notice gender differences by demographic group and percent of respondents who did not notice race differences by demographic group. Percentages are calculated using the total number of respondents (see Table 2) in each group from our data set 


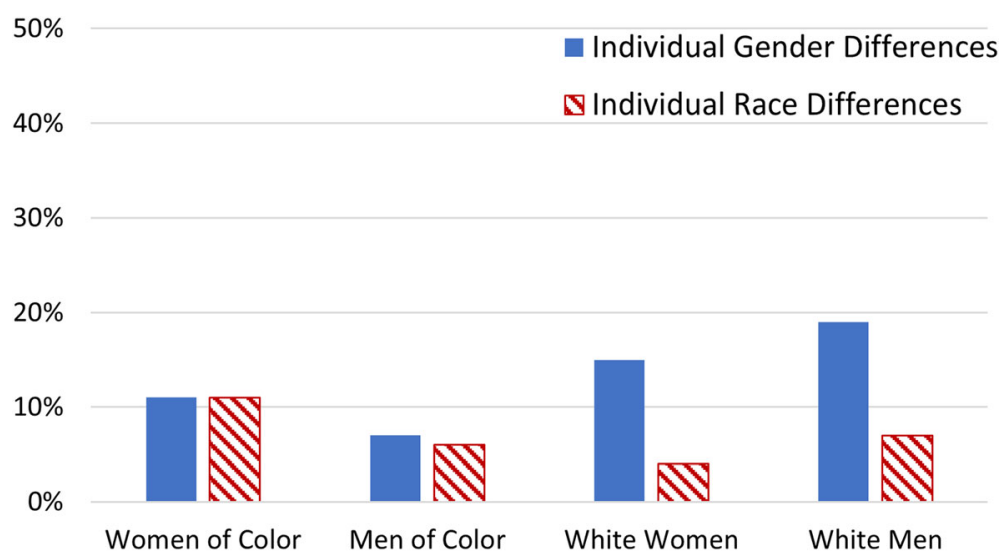

Fig. 3 Percent of respondents who cited individual characteristics as being the cause of gender and race differences in STEM experiences by respondents' own race-by-gender category

involved the idea that women are more academically motivated than their peers who are men:

I feel like guys like to take the easier route and it's not a lot of guys that like to be challenged, and for a female it's kind of like they will work harder instead of taking the easier route.-Black woman biology major

\section{Differences in individual characteristics of different races}

Some respondents described individual characteristics of different races as the contributor to racial differences in experiences in STEM without inference to these characteristics being driven by racism. Statements were coded in this category when the interviewee gave no indication of larger systemic causes to the differences between races (i.e., it is just Asian culture). The most common of these was the belief that some races value science more. In particular, many students mention people from Asian descent come from cultures that encourage the pursuit of science. As one Black woman majoring in biology stated "Not every African American would want to be a doctor, some people want to pursue other careers and same thing with Caucasians and Asians. I think there's a larger number of Asians who go into the math and science and technology kinda stuff than other races." And as a White male majoring in computer science expressed "I can see it being encouraged a lot in the Asian communities because they put such a focus on both successful careers and like math-oriented careers."

Finding three: Some students notice differences in STEM experiences due to sexism/racism

We now turn to those who attributed impacts to structural or cultural systems (i.e., sexism and racism). As mentioned above, if a student listed any impact that could be categorized this way, they were counted in this category. Therefore, this category represents the students who noted any sexism or racism impacts even if they also noted non-sexism and racism impacts. (We note that the terms "sexism" and "racism" were rarely used explicitly by students.) Under this broad category, student responses were categorized into three main categories (impacts due to minority status, impacts due to discrimination, and, for race only, impacts due to social and cultural capital). Each of these is described in detail below for both gender and race.

\section{Impacts related to being one of the few}

A number of responses were coded as describing impacts related to the underrepresentation of some groups. For both gender and race, respondents talked about women and students of color feeling intimidated, feeling pressure to work harder, and feeling out of place due to their underrepresentation. Responses related to gender and race tended to be similar; therefore, we combine our discussions of gender and race below. Figure 4 presents the number of students who fell into this broad category for gender and race impacts. A description of the most common impacts attributed to the minority status of women and people of color in STEM are described in detail below.

Underrepresentation of women and people of color in STEM leads to feelings of intimidation A common theme under the impacts due to being a member of an underrepresented group was that those in the minority feel intimidated due to their underrepresented status, either directly when pursuing their degree or in job markets or internships. Often this came up as an expression of lacking the confidence to pursue STEM or of feeling discouraged. For example, when discussing her math department, one White woman majoring in mathematics 
said "I have been surrounded by several males and sometimes I have gotten intimidated by it just because there are only six [female students] all together being outnumbered by these males... Sometimes I do get intimidated myself." Nearly all responses in this category for gender were given by women, with White women making up the majority.

Women and students of color feel pressure to work harder due to their visible status In the previous section we identified the belief that women naturally work harder than men as a reason given for gender differences in STEM. Relatedly, some students mentioned that due to low representation of women and people of color in STEM, women, and students of color would feel pressure to work harder. Although superficially similar (women work harder), these two categories are distinctly different due to the mechanism proposed (individual vs. structural) and are therefore reported separately. In a later section this same theme of working harder surfaces again in relation to bias.

Frequently, responses about women and people of color working harder were related to disproving stereotypes about women and people of color in science and feeling a need to prove themselves in the field. For example, one White woman double majoring in mathematics and computer science said "It feels strange if I'm the only female in a class... it feels like I have to keep up... like, if I'm not as good, then it says something bad." Another White woman majoring in biology spoke of pressure due to stereotypes as well, stating "It makes me feel like there is more pressure because I feel like if I don't do as well or better that it will be like, turned into a gender thing... I don't want to be treated differently... I want to kind of prove myself like I'm not only as good, I'm better than you guys."
After discussing the low numbers of women in engineering, one White man majoring in aerospace engineering expressed feeling sorry for women in engineering because they would be "out of their comfort zone" to be in a class of all men. He went on to say "I guess that [women] would have to work a little bit harder, I would think, to prove themselves."

Many people said that students of color likely feel pressure to work harder due to their low representation in STEM. Much of these comments had to do with combating or disproving stereotypes about certain races, such as Black and Hispanic, in STEM. For example, one Hispanic man majoring in engineering technology said "I kind of push myself a little bit more just because ... the majority \{in STEM\} are White, White males, so being the only Hispanic in my class kinda makes me wanna try a little bit harder... I wanna be seen as equally competent as a White male." One Black man majoring in mathematics expressed feeling there was "equal opportunity" in STEM, but also remarked: "I have the drive to try to prove others wrong and I'm here in this field and I'm successful in this field so far and I'm just going against the stereotype and against the statistics."

We note that what these students are describing an experience of stereotype threat (Steele, 1997). Stereotype threat research demonstrates that a person whose group is numerically marginalized in a classroom may perceive a spotlight on their "performance." If there is also a negative cultural stereotype associated with their group, the anxiety that spotlight triggers, combined with the negative cultural stereotype, can lead to diminished effort and lower performance. Students from underrepresented groups may decline to try hard so any "failures" in the class are due to their diminished effort rather than confirmation of the stereotype. The widespread

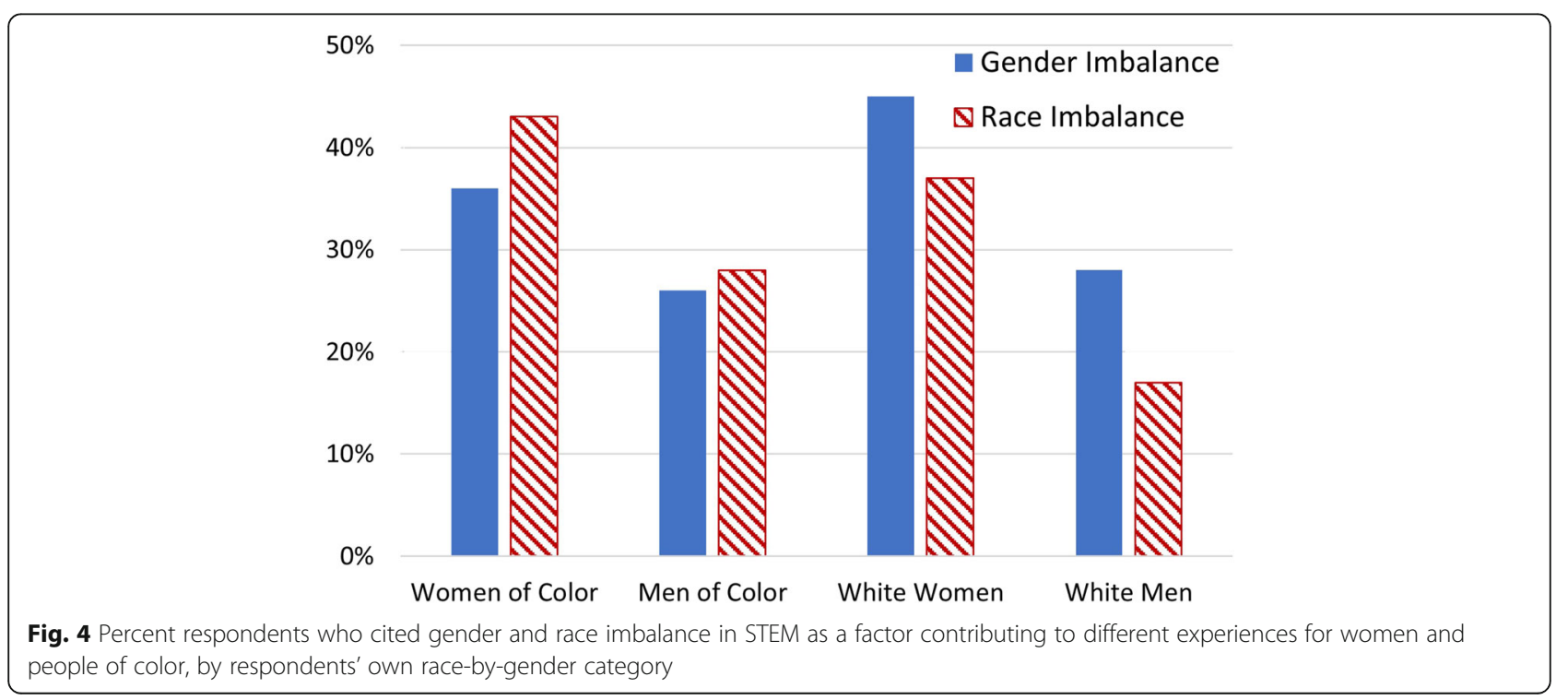


stereotype threat associated with certain racial/ethnic and gender groups has been demonstrated as a barrier to success for some underrepresented groups in STEM. Importantly, a number of our respondents indicate that they fight the stereotype and try harder, rather than diminish their efforts.

Women and people of color feel out of place Many students speculated that because women are outnumbered in STEM, they may feel out of place. For example, one White women majoring in computer science said about her major, "I know it's easy for women to feel out of place or alienated just because it's not a very popular profession for women." Many women voiced this feeling after being directly asked if they had ever felt out of place in their degree program. For example, one Hispanic woman majoring in computer science said "Sometimes [I feel out of place] because I am pretty much the only girl, I think in all of my classes this semester." When asked if the feeling had changed over time, she said "no" and continued "I think from the very beginning everyone is always like, 'oh a girl in computer science-- this never happens' so I am probably used to it at this point."

Although no White men expressed such views, some participants either experienced or observed that low representation of certain races and disproportionate representation of others can make students of color feel out of place. One Black woman spoke from her direct experience as a Black woman in biology, "Sometimes I feel out of place because I'm black and that's a minority in biology." Another White woman spoke from experience as well, but spoke from a place of knowing an AfricanAmerican man in her biology program, "I remember there was like one African-American in our class and I always felt like, bad for him because I was like oh, he's like the only one... He kind of felt uncomfortable and he made a couple comments about [how] he felt alone." Her acquaintance felt uncomfortable and alone because he was the sole Black person in the class. Similarly, a Hispanic woman majoring in computer science expressed discomfort because she was the only Hispanic person in her statistics class, "I was very uncomfortable... It was just mostly because I think I was maybe the only Hispanic in that class... I would always sit like in the back of the classroom so that like nobody would notice me... And in my other classes, I would always just stay toward the front of it."

\section{Notices impacts of sexism/racism as discrimination}

Students mentioned impacts that can be characterized as forms of discrimination against women or people of color. Most responses in this category either related to employment discrimination in STEM (finding employment and/or being paid fairly) or in cultural bias in favor of men and White people based on the stereotype that men and White people have more ability in STEM, or are expected and encouraged to pursue STEM. Figure 5 below indicates the number of students coded as expressing belief in discrimination as likely impacts of such stereotypes. The following sections discuss the various subcategories of discrimination.

Employment discrimination against women and people of color Some students reported the belief that the employment experience likely is different for women and people of color. Responses acknowledged women and people of color can experience discrimination when seeking a job in STEM or in terms of being paid fairly. For example, an Asian female chemistry major said:

I think that out in the professional world there is still a bias towards men and against women when it comes to the science type field. It doesn't matter the degree you got, where you got it from, or the grades. I think that when it comes to science they are still going to prefer the male over the female. Why, I don't know. I feel like the women have to work harder and get more experience in order to get the job.

A Black female information technology major echoed the difficulties of getting a job and also mentioned unfair pay. "I have heard different stories that men get paid more than women... I have heard that men actually get paid more and women have a harder time trying to put themselves out there and trying to prove themselves when you are competing against men in the IT field."

The idea that there is discrimination in employment was most commonly associated with gender-based discrimination with only a few students mentioning it regarding race.

Bias in favor of men or White people Many students identified aspects of bias in STEM either in favor of men and White people or against women and people of color. Typically this was in the form of assumptions made in society at large about the competencies of women and people of color in STEM or of the expectations of careers women and men pursue. Students very commonly added that when faced with bias, women and/or people of color felt a need to work harder to prove themselves against these societal expectations.

Assumptions of ability Numerous students cited the stereotype that men and White people are more competent in STEM fields. For example, a Black female biology major stated "I feel like our male teachers don't really expect females to do well with the subject; they kind of think that males are just all knowing and all seeing 


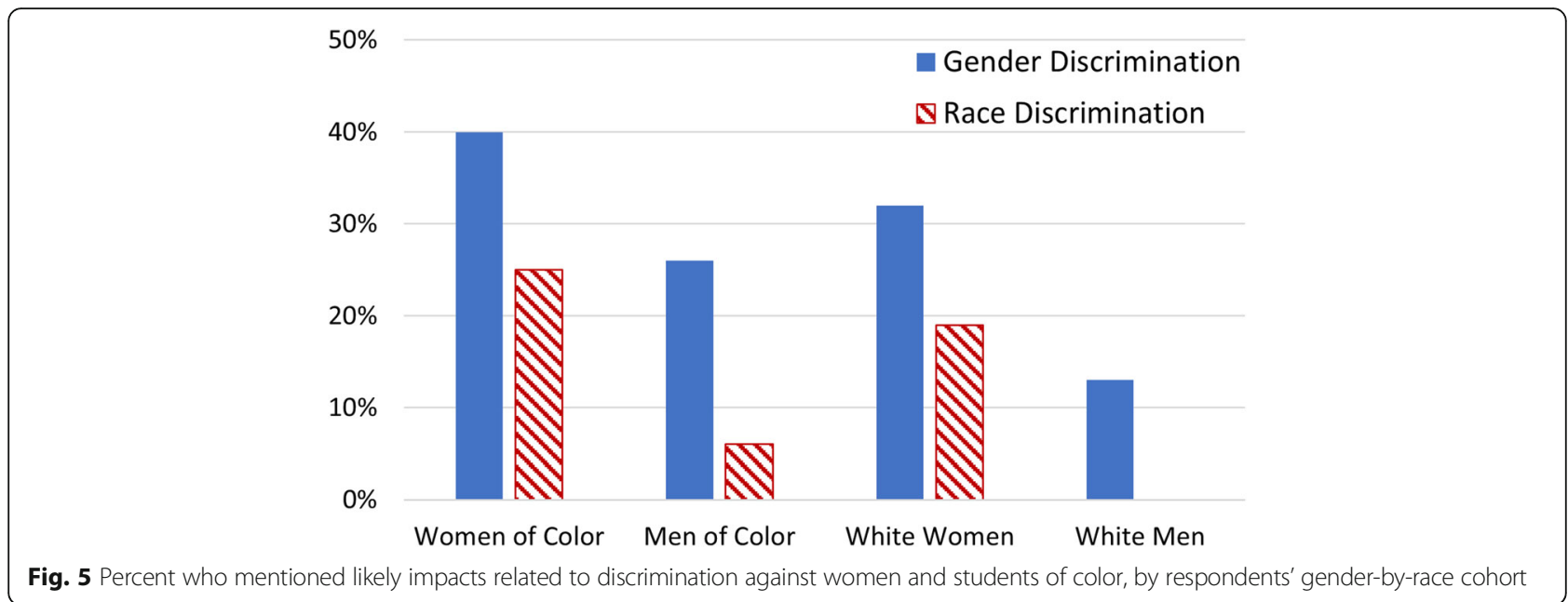

everything. I feel like sometimes they think we are not capable of doing certain things." Or as another Black woman majoring in chemistry stated: "I've had some professors who make it plain and clear that, no, because you're Black you're not focused and I'm not really trying to, you know, give you the time of day." We note that in every case where a specific race was mentioned as being perceived to have less ability, Black people were the target.

Interviewees sometimes attributed the perception about differing abilities to stereotypes about who does science. When speaking about race students frequently referred to stereotypes about Asians and Indians being more science inclined, for example a White man who left engineering stated, "I know there are probably some cultural stereotypes that favor the Asian being known for math and engineering and especially now in today's world and also the traditional you know Caucasian engineers as your traditional stereotype."

Assumptions about who pursues science There were a number of comments indicating a bias in the expectation that STEM is for men and White people to pursue. As a Hispanic woman majoring in geology expressed, "I don't think that there is intentional racism. I think that it is probably just seen as more of a White field." Many students indicated that the bias toward men and White people resulted in women and students of color being explicitly discouraged from pursuing STEM. As a White male majoring in computer science expressed "Women just aren't encouraged to make such a career choice growing up. I mean it's totally fine if dudes sit on the computer all day and play video games and stuff like that, which for our generation is easily one of the bigger influences on computer scientists but it's not okay for girls to do it... they are losers if they sit around and play video games."

White students have greater social and cultural capital One emergent theme unique to discussions of race had no parallel for gender: social and cultural capital (Bourdieu, 1990; Lareau, 2011). Social and cultural capital refers to resources available to students, often linked to their higher socioeconomic status. Social and cultural capital can include superior high school preparation (higher quality teachers, AP courses), parental or extended family experiences with STEM subjects and occupations (role models), informal family and community STEM learning (science museums, summer camps, parental assistance with STEM homework), prior knowledge about applying to and succeeding in college (parents' education), informal networks as resources, and encouragement from peers or family. For example, when asked if there are race differences in pursuing a STEM degree, one Black woman who left a chemistry major said:

Yes, and mainly because [my school] is in a poverty stricken area. I think people from like places where it's more financially secure and stuff they have a better advantage over students with like poverty backgrounds, which would be like the Native American students or some of the Black students here, but with that being said... I think if you put in the same amount of work I don't think you would have a problem with that disadvantage.

Another Black woman majoring in biology discussed socioeconomic status and how the occupation of your parents can influence your pursuit of a STEM degree. She spoke of her friends' "richer families" with parents 
who were lawyers and doctors, whereas her parents were a truck driver and social worker. She then said "I didn't really know anything about science also maybe because of that because they \{my parents\} didn't have those types of jobs."

Students of color and White women were the only students who mentioned something related to social or cultural capital as an explanation for disparate lived experiences as a STEM student. None of the White men we interviewed made connections between race and social or cultural capital in their reflections on the STEM experience.

Bias results in women and students of color having to work harder Commonly, when bias was identified, students said that a consequence of this was that women and students of color had to work harder to prove themselves. For example, a white female biostatistics major stated "I think sometimes you have to work a little harder to be taken seriously I think as a blonde female." Similarly, a black female biology major expressed, "I think that is mostly a male-dominated field so I feel that women that do choose to go into this career have to work harder to prove that they are as qualified."

We note that the notion that women and students of color work harder was common among all of our codes. We elaborate on this finding later in the article.

\section{Finding four: Some students perceive that} underrepresented students benefit due to increased opportunities for scholarships and jobs

Approximately 3\% of students (most of whom were White women) felt that women and students of color had advantages over men and White students because of the current cultural and political emphasis on expanding STEM undergraduate populations to those groups previously underrepresented.

Comments about women and students of color benefiting from their demographic profiles were often related to job prospects, scholarship opportunities, and general encouragement. For example, a Black woman majoring in electrical and computer engineering commented on the experience of being both a woman and a racial minority and the benefits that would come along with that identity in the job market. She spoke about companies needing to meet a "minority quota" and how she was both female and African-American. She went on to say "I've gone to career fairs and walked by a booth and a guy is going 'no, no,... come here, come here, we're looking for African American females' and I don't know anything about their company and I might get an interview."
Another common theme focused on underrepresented groups' receiving extra encouragement. As articulated by a White woman majoring in mathematics:

\section{I feel like as a woman at [my university] I have been especially encouraged and I don't know if that's just my experience but I feel like teachers are so afraid to be sexist that they sort of over put the emphasis on helping the women in their class.}

White women were the most likely to comment on how people of color, a group they are not a part of, benefit from their race. However, they were also the most likely to comment on themselves being able to benefit due to their gender.

While there is much discourse about programs to encourage underrepresented groups to pursue STEM, including scholarship opportunities specific to these groups, the available data (see literature review section above) suggest large and negative impacts on these groups that limit access and ability to succeed. Even when ignoring factors that contribute to a general chilly climate for underrepresented groups and focusing only on the hiring process there still is no evidence that women or people of color are advantaged (Ceci and Williams, 2015).

\section{Finding five: Women/students of color are commonly perceived as working harder than men and White students}

As discussed above, a common theme throughout our various interviews was the belief that women and/or students of color work harder as a result of their demographic group's marginalized status. Figure 6 shows the distribution of this perception by demographic. Sometimes this was attributed to a characteristic of a group without an attribution to any cultural or systemic factors that marginalized the group. Other times, the theme of extra effort by women and students of color appeared as an expression of feeling pressure to do well as a result of being unusual or standing out. The third category in which we saw this theme arising was in students' expressed belief that women and students of color felt a need to prove themselves or work extra hard to do better than their white/male peers due to a perceived bias against them. As we have seen before, White men were the least likely to report this impact and respondents were more likely to report the impact for women than for students of color.

We note that a number of students observed the impact of needing to exert more effort had on the mental state of a student, either allowing men to be more relaxed, or resulting in women and students of color being more stressed. For example, a White woman majoring in 


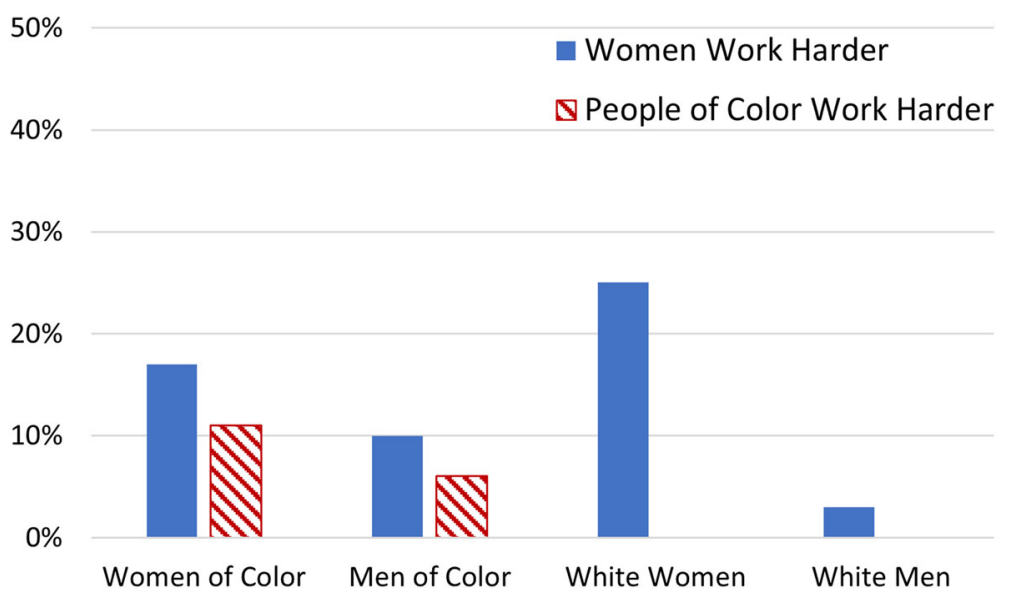

Fig. 6 Percent respondents who expressed theme that women and people of color work harder, by respondents' own race-by-gender category. We note that no student indicated that men or White students work harder than women or students of color

civil engineering noted her perception that men were more relaxed, "I think it's more challenging for women... just looking at the guys they seem pretty set and you know they're going to pass and they're going to get a job, it seems pretty laid back. Whereas you see the women in civil engineering and they are really working hard and striving to outdo the guys because there's just such a stigma." Another white female chemistry major noted the extra stress this places on women "I feel like with women ... in chemistry are trying to prove themselves and push to show that they are able and capable of handling the workload and doing the work... I would say just in looking at the men and women in my major, the women are more ...I don't want to say stressed out but like they are more ...meticulous and anxious and organized about things whereas the men are more relaxed."

We highlight this finding because it has important implications. At no time did we ever ask a direct question that would elicit the expression of women or students of color feeling a need to exert more effort. Yet, a significant number of students brought this up on their own. Most commonly they attributed extra effort from women and students of color to a desire to prove themselves because of bias against them and noted it leads to significant stress.

Chronic stress is associated with poor mental health (including anxiety, depression, and mood disorders) as well as lower learning outcomes (Vogel and Schwabe, 2016). The literature on mental health of STEM students relative to their demographic is sparse but consistent with our findings that women and students of color report more behavior associated with stress. Studies indicate women in STEM have lower overall mental health (Deziel, Olawo, Truchon and Golab, 2013), more anxiety
(Saravanan and Wilks, 2014), and more depression than men (Kotok, 2007). In addition to the impacts of chronic stress on women and students of color in STEM, there is also evidence (Smith, Lewis, Hawthorne and Hodges, 2013) that the perception of having to work harder than men in STEM leads to women to be less likely to feel they belong and to be less motivated to pursue STEM.

Yet, many of our participants identified their extra effort as a badge of honor. For example, a Black male mathematics major explained "I have the drive to try to prove others wrong and I'm here in this field and I'm successful in this field so far and I'm just going against the stereotype and against the statistics."

\section{Discussion}

A strength of this study lies in the intersectional analysis of perceptions of racism and sexism in college STEM experiences. Unlike prior research about each of the demographic groups in our study, our findings permit us to compare and contrast perceptions of racism and sexism among members of several race-by-gender cohorts of college seniors. In summary, we found the following:

- Undergraduate students are often unaware that gender or race impacts the experiences of students in STEM despite extensive research indicating they have all experienced such impacts.

- Students who are aware of impacts frequently attribute the effects to differences between the genders and people of different races and not to any systemic or cultural causes.

- Among students who were aware of differences, the most common examples given were that men and some races are more interested in science (and 
therefore more likely to pursue and persist) and have different work ethics.

- Some students who are aware of impacts attribute them to sexism and/or racism, though none used these terms explicitly. Most commonly, respondents state that being a minority can lead to students feeling intimidated, feeling pressure to work harder, or feeling out of place, and these feelings have a negative impact on their educational experience. Students also identify ways women and students of color are discriminated against, especially in relation to experiences in employment and in bias against them.

- Differing levels of social and/or cultural capital were cited as an explanation for why some races are more represented than others. In doing so, students often conflated race with social class differences in opportunities to prepare for STEM.

- A small number of students, mostly White women, believe women and students of color benefit from their underrepresented status in STEM due primarily to societal efforts encouraging more women and students of color to pursue STEM.

- The belief that women and students of color work harder in STEM appeared across categories including the following: harder work is a characteristic of the group, it is due to being a minority, and it is due to discrimination.

Overall, our findings can be broken down into three broad categories: (a) respondent does not notice differences, (b) respondent notices internal differences between groups (non-sexism/racism), and (c) respondent notices differential experiences and identifies them as grounded in structural factors (sexism/racism).
Differences are summarized in Fig. 7. Below, we highlight and discuss two important trends in our findings.

\section{Except for women of color, students were less likely to perceive race has an impact}

Most students reported perceiving fewer impacts due to race than to gender. We note that the research literature indicates that both race and gender significantly impact students' experiences in STEM. Our data do not allow for explanations of this gap in perceived impacts. We note that the students we interviewed were attending schools with a racially diverse population (the overall system is approximately $40 \%$ students of color). This may affect their awareness of race impacts compared to students in other areas of the country where students of color are less represented at their universities.

We suspect that the enhanced awareness of gender impacts may be due the greater attention given to issues of women in STEM in the media. It may be that students have simply had their attention directed more to issues of gender in STEM than race. However, it is beyond the scope of this study to investigate this possible reason. The finding of lower awareness of race impacts than gender impacts suggests there is a need to raise awareness of the influence of race, racism, and overall racial climate for students pursuing a STEM degree. It is more difficult to address inequity if there is not widespread recognition of its existence.

White males were the least likely to perceive impacts of race or gender on the experience of majoring in STEM We note that White men were the least likely to recognize race or gender impacts on students' experiences in STEM. This finding is very illuminating. White men are the majority population in many subfields of

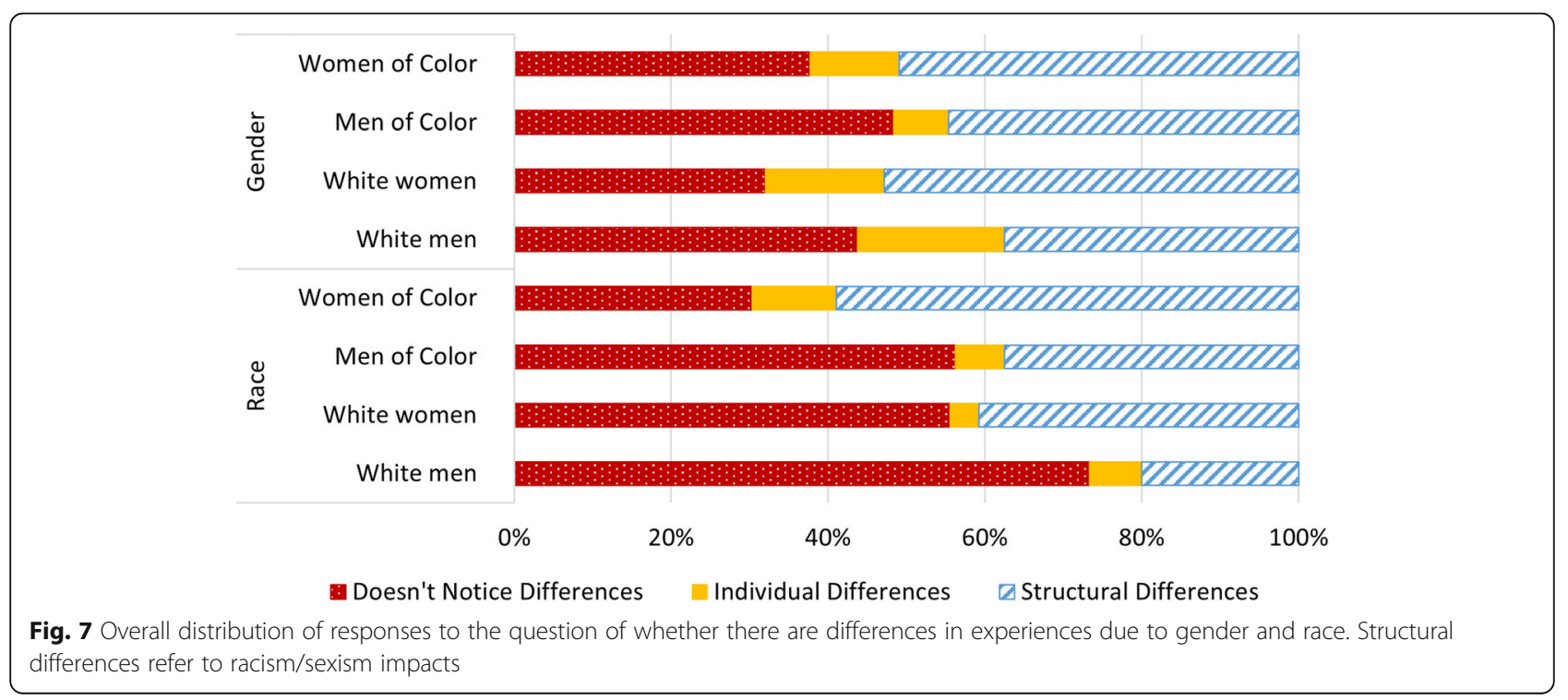


STEM and are overrepresented in positions of power and influence in STEM (i.e., they are more likely to achieve tenure and fill leadership positions). Only $40 \%$ of White men in our sample acknowledged any impact of sexism in STEM and only $25 \%$ acknowledged any impact of racism on the experience of pursuing a STEM major. The majority of White men appear to be unaware of the ways in which gender and race shape both their own and others' experiences. If the majority of individuals in the dominant demographic group do not recognize the disparate experiences of other demographic groups, it will be difficult to create the disciplinary cultural and structural conditions necessary to foster greater equity.

Research around equity typically focuses on the experiences of those who are in the minority rather than those in the majority. This implicitly views inequity in the context of deficits in individuals in minority populations rather than in the context of the structures and culture created by the majority population. Efforts that focus on people from underrepresented groups are likely limited in their impact on equity because the ideas and practices of the majority population also need to be understood, challenged, and changed.

\section{Implications and future research}

We offer the following recommendations for future work:

1. Efforts to address inequality in STEM should not disregard White men. As discussed above, our analysis indicates the majority population in STEM is unaware of the experiences of marginalized groups. Lasting systemic change will necessitate research to document, challenge, and leverage their perspectives. As others are increasingly noting (Fox, Sonnert and Nikiforova, 2009; Malcom and Malcom, 2011), reform efforts often operate from a deficit model, assuming the marginalized population needs to change in order to achieve success (i.e., mentoring programs, enrichment activities, scholarships). This perspective disregards the impacts of the majority population by locating the problem and solution with marginalized groups. Additionally, this perspective devalues the perspectives of the marginalized group in favor of the norms of the privileged group, without questioning the value of the norms. We argue the focus on White men in STEM as the norm to be achieved, while disregarding them as a point of change, is a shortcoming that needs to be addressed. If the group that most influences the dominate culture is unaware of that culture or its impacts on others, and if little effort is put on identifying and changing the dominant group's understanding, by what mechanism would change be expected?

2. Greater attention is needed to issues of race in STEM. We note there was less awareness of race impacts. We hypothesize this is most likely due to a lack of awareness rather than actual experience, as much of the discourse among STEM research has focused on the experiences of women in STEM. There is ample evidence that race has a significant impact on one's experience in STEM (National Science Foundation, 2019; Ong et al., 2011) and no indications that it is less than the impact due to gender. We argue that inequity can be better addressed by a greater acknowledgement of the inequities experienced based on race.

3. More work is needed on the relationships among racism, sexism, and the stress they trigger among underrepresented groups. As discussed, a large number of students commented that those who are marginalized work harder, which was often connected to feeling more stress and anxiety in response to marginalization. We found little literature addressing what appears to be a common and impactful issue. We argue that this is an important and under studied area.

\section{Limitations}

In the work presented here, our sample is not representative along several dimensions. It is not geographically diverse (all students were from North Carolina), is limited in socioeconomic diversity (all students were attending a public institution), and was subject to selfselection bias. Additionally, due to the problematic nature of dividing the sample into overly descriptive categories, findings must be applied at a general level. So, for example, we cannot offer comparisons between different non-White racial groups, or between students pursuing different majors within STEM. And finally, we did not account for other intersectional identities that likely influence beliefs (LGBTQ+ identities, for example).

\section{Conclusion}

Prior research has identified numerous ways women and people of color have experiences as they pursue STEM that limit their access and engagement. Our analysis of the perceptions of race and gender impacts in STEM identified the relative lack of awareness of these discouraging experiences among a large portion of White male undergraduates. The differing perceptions of the least privileged students (women of color) and the most privileged (White men) is consistent with the idea of STEM as White male property. Within STEM, the needs, viewpoints, and contributions of White men are prioritized, 
signifying a culture of White male ownership and power. White men have little need or motivation to attune to the experiences of women and students of color, as these experiences are outside the ownership of STEM.

White male undergraduates' lack of awareness of what their peers experience becomes part of the "chilly climate" with which women and students of color must contend en route to their STEM degrees and communicates that they perceive White male ownership of the field. Addressing the deficiency in awareness, and the underlying culture that supports the lack of awareness, must be part of any effort to address equity in STEM. Equity in STEM extends beyond individuals striving for their degrees. Greater equity in STEM fields can begin to address the loss of talent among historically excluded groups and the issues of distributive [in]justice related to STEM careers and social mobility.

\section{Acknowledgements}

We would like to thank Mette Evelyn Bjerre, Savannah Larimore, Lauren Valentino, and Aaron Wood for conducting interviews and assisting with coding. We also thank Martha Cecilia Bottia for conducting interviews.

\section{Authors' contributions}

$\mathrm{MD}$ contributed to the conception and design of the study; led the initial draft of the data codebook; participated in data collection, analysis, and interpretation; and led writing and revising of the manuscript. KR contributed to the analysis and interpretation and contributed equally with $\mathrm{MD}$ to writing and revising of the manuscript. RM contributed to the conception and design of the study; contributed to data collection, analysis, and interpretation; and contributed to the writing and revising of the manuscript. ES contributed to the conception and design of the study; supervised execution of data collection; contributed to analysis and interpretation; and contributed to the writing and revising of the manuscript. SM contributed to the conception and design of the study and contributed to data collection, analysis, and interpretation. The authors read and approved the final manuscript.

\section{Authors' information}

The authors are all White, cisgendered women. Two of the authors (MD and KR) are academically trained in both physics and education. Three of the authors (ES, RM, and SM) are academically trained in sociology.

\section{Funding}

The authors gratefully acknowledge support provided by the National Science Foundation award numbers DUE-0969286, DRL-1420363, and DRL1420350. Any opinions, findings, and conclusions or recommendations expressed in this material are those of the authors and do not necessarily reflect the views of the National Science Foundation. Publication of this article was funded by the University of Colorado Boulder Libraries Open Access Fund.

\section{Availability of data and materials}

Data was collected via interviews which could be personally identifiable. Sharing of collected data was not approved by the Institutional Review Board.

\section{Competing interests}

The authors declare that they have no competing interests.

\section{Author details}

'Department of Physics, University of Colorado Boulder, UCB390, Boulder, CO 80309-0390, USA. ${ }^{2}$ Department of Sociology, University of North Carolina at Charlotte, 9201 University City Blvd, Charlotte, NC 28223, USA.
Received: 17 November 2019 Accepted: 15 September 2020

Published online: 12 October 2020

\section{References}

Annamma, S. A. (2015). Whiteness as property: Innocence and ability in teacher education. Urban Review, 47(2), 293-316.

Bonilla-Silva, E. (2006). Racism without racists: Color-blind racism and the persistence of racial inequality in the United States. Rowman \& Littlefield Publishers.

Bourdieu, P. (1990). The logic of practice. Translated by Richard Nice. Stanford: Stanford University Press.

Bowleg, L. (2008). When Black+ lesbian+ woman‡ Black lesbian woman: the methodological challenges of qualitative and quantitative intersectionality research. Sex Roles, 59(5-6), 312-325.

Ceci, S. J., \& Williams, W. M. (2015). Women have substantial advantage in STEM faculty hiring, except when competing against more-accomplished men. Frontiers in Psychology, 6, 1532.

Collins, P. H. (2015). Intersectionality's definitional dilemmas. Annual Review of Sociology, 41, 1-20.

Committee on Underrepresented Groups and the Expansion of the Science and Engineering Workforce Pipeline (2011). Expanding underrepresented minority participation: America's science and technology talent at the crossroads. Washington, DC: National Academies Press.

Crenshaw, K. (1990). Mapping the margins: Intersectionality, identity politics, and violence against women of color. Stan. L. Rev., 43, 1241.

Crosby, F. (1984). The denial of personal discrimination. American Behavioral Scientist, 27(3), 371-386.

Deziel, M., Olawo, D., Truchon, L., \& Golab, L. (2013). Analyzing the mental health of engineering students using classification and regression. In Educational Data Mining 2013.

Fisher, A. J., Mendoza-Denton, R., Patt, C., Young, l., Eppig, A., Garrell, R. L., ... Richards, M. A. (2019). Structure and belonging: Pathways to success for underrepresented minority and women PhD students in STEM fields. PLoS One, 14(1), e0209279.

Fox, M. F., Sonnert, G., \& Nikiforova, I. (2009). Successful programs for undergraduate women in science and engineering: Adapting versus adopting the institutional environment. Research in Higher Education, 50, 333-353.

Funk, C., \& Parker, K. (2019). Women and men in STEM often at odds over workplace equity. Retrieved from https://www.pewsocialtrends.org/2018/01/ 09/women-and-men-in-stem-often-at-odds-over-workplace-equity/

Gee, B., \& Peck, D. (2017). The illusion of Asian success. Ascend: Pan-Asian Leaders. https://cdn.ymaws.com/www.ascendleadership.org/resource/resmgr/ research/ThelllusionofAsianSuccess.pdf.

Haney-López, I. (2006). White by law: The legal construction of race (10th anniversary ed.). New York: New York University Press.

Harris, C. (1995). Whiteness as property. In K. Crenshaw, N. Gotanda, G. Peller, \& K. Thomas (Eds.), Critical race theory: The key writings that formed the movement, (pp. 276-291). New York: The New Press.

Hill, C., Corbett, C., \& St Rose, A. (2010). Why so few? Women in science, technology, engineering, and mathematics, (p. 20036). Washington, DC: American Association of University Women.

Jost, J., \& Hunyady, O. (2003). The psychology of system justification and the palliative function of ideology. European Review of Social Psychology, 13(1), 111-153.

Knowles, E. D., Lowery, B. S., Chow, R. M., \& Unzueta, M. M. (2014). Deny, distance, or dismantle? How White Americans manage a privileged identity. Perspectives on Psychological Science, 9(6), 594-609. https://doi.org/10.1177/ 1745691614554658.

Kotok, A. (2007). Depression in the scientific and technical workforce. Retrieved from https://blogs.sciencemag.org/sciencecareers/2007/10/depression-in-t.html

Ladson, B., \& G. \& Tate, W. (1995). Toward a critical race theory of education. Teachers College Record, 97(1), 47-68.

Lareau, A. (2011). Unequal childhoods. Race, class, and family life. Berkeley: University of Calif Press.

Malcom, L., \& Malcom, S. (2011). The double bind: The next generation. Harvard Educational Review, 81(2), 162-172.

Mickelson, R. A., Parker, A., Stearns, E., Moller, S., \& Dancy, M. (2015). Family matters: familial support and African American female success. In Ruiz-Smith, \& S. L. Clark (Eds.), Contemporary African American Families: Achievements, Challenges, and Empowerment Strategies in the 21st Century. New York: Routledge-Taylor \& Francis. 
Miller, C. W., Zwickl, B. M., Posselt, J. R., Silvestrini, R. T., \& Hodapp, T. (2019). Typical physics Ph. D. admissions criteria limit access to underrepresented groups but fail to predict doctoral completion. Science Advances, 5(1).

Moller, S., Banerjee, N., Bottia, M. C., Stearns, E., Mickelson, R. A., Dancy, M., et al. (2015). Moving Latino/a students into STEM majors in college: the role of teachers and professional communities in secondary schools. Journal of Hispanic Higher Education, 14(1), 3-33.

Moore Mensah, F., \& Jackson, I. (2018). Whiteness as property in science teacher education. Teachers College Record, 120(1), 1-38.

National Science Foundation, National Center for Science and Engineering Statistics (2019). Women, minorities, and persons with disabilities in science and wngineering: 2019, (pp. 19-304). Alexandria: Special Report NSF Available at https:/www.nsf.gov/statistics/wmpd.

NCCJ: National Conference for Community and Justice. (2018). What is privilege? Retrieved from https://nccj.org/what-privilege

Norton, M. I., \& Sommers, S. R. (2011). Whites see racism as a zero-sum game that they are now losing. Perspectives on Psychological Science, 6(3), 215-218.

Ong, M., Wright, C., Espinosa, L., \& Orfield, G. (2011). Inside the double bind: A synthesis of empirical research on undergraduate and graduate women of color in science, technology, engineering, and mathematics. Harvard Educational Review, 81(2), 172-209.

Rainey, K., Dancy, M., Mickelson, R., Stearns, E., \& Moller, S. (2018). Race and gender differences in how sense of belonging influences decisions to major in STEM. International Journal of STEM Education, 5(1), 10.

Rainey, K., Dancy, M., Mickelson, R., Stearns, E., \& Moller, S. (2019). A descriptive study of race and gender differences in how instructional style and perceived professor care influence decisions to major in STEM. International Journal of STEM Education, 6(1), 6.

Rugheimer, S. Women in STEM resources. (2019) Retrieved from http://www. sarahrugheimer.com/Women_in_STEM_Resources.html

Saravanan, C., \& Wilks, R. (2014). Medical students' experience of and reaction to stress: the role of depression and anxiety. The Scientific World Journal, 2014.

Smith, J. L., Lewis, K. L., Hawthorne, L., \& Hodges, S. D. (2013). When trying hard isn't natural: Women's belonging with and motivation for male-dominated STEM fields as a function of effort expenditure concerns. Personality and Social Psychology Bulletin, 39(2), 131-143.

Stearns, E., Bottia, M. C., Giersch, J., Mickelson, R. A., Moller, S., Jha, N., \& Dancy, M. (2019). Do relative advantages in STEM grades explain the gender gap in selection of a STEM major in college? A multimethod answer. American Educational Research Journal. https://doi.org/10.3102/0002831219853533.

Steele, C. M. (1997). A threat in the air: How stereotypes shape intellectual identity and performance. American Psychologist, 52(6), 613-629.

Syracuse University. (2018). Impact of marginalization. Retrieved from http:// counselingcenter.syr.edu/social-justice/impact-of-marginalization.html

Tropp, L. R., \& Barlow, F. K. (2018). Making advantaged racial groups care about inequality: Intergroup contact as a route to psychological investment. Current Directions in Psychological Science, 27(3), 194-199.

Vogel, S., \& Schwabe, L. (2016). Learning and memory under stress: Implications for the classroom. npj Science of Learning, 1, 16011.

Williams, J. C., Li, S., Rincon, R., \& Finn, P. (2016). Climate control: Gender and racial bias in engineering. Center for WorkLife Law. UC Hastings College of the Law.

\section{Publisher's Note}

Springer Nature remains neutral with regard to jurisdictional claims in published maps and institutional affiliations.

\section{Submit your manuscript to a SpringerOpen ${ }^{\circ}$ journal and benefit from:}

- Convenient online submission

- Rigorous peer review

- Open access: articles freely available online

- High visibility within the field

- Retaining the copyright to your article

Submit your next manuscript at $\boldsymbol{\nabla}$ springeropen.com 